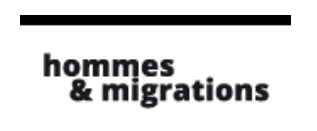

\section{Hommes \& migrations}

Revue française de référence sur les dynamiques

migratoires

$1317-1318 \mid 2017$

L'Europe en mouvement

\title{
La mobilité étudiante en Europe. Une lente institutionnalisation sans réelle démocratisation
}

\section{Magali Ballatore}

\section{OpenEdition}

\section{Journals}

Édition électronique

URL : http://journals.openedition.org/hommesmigrations/3877

DOI : 10.4000/hommesmigrations.3877

ISSN : 2262-3353

\section{Éditeur}

Musée national de l'histoire de l'immigration

\section{Édition imprimée}

Date de publication : 1 avril 2017

Pagination : 79-86

ISBN : 978-2-919040-38-4

ISSN : $1142-852 X$

\section{Référence électronique}

Magali Ballatore, «La mobilité étudiante en Europe. Une lente institutionnalisation sans réelle démocratisation », Hommes \& migrations [En ligne], 1317-1318 | 2017, mis en ligne le 01 avril 2020, consulté le 04 janvier 2020. URL : http://journals.openedition.org/hommesmigrations/3877 ; DOI : 10.4000/hommesmigrations.3877 


\title{
LA MOBILITÉ ÉTUDIANTE EN EUROPE. UNE LENTE INSTITUTIONNALISATION SANS RÉELLE DÉMOCRATISATION
}

Par MAGALI BALLATORE, maître de conférences en sociologie, LAMES, CNRS, Université Aix-Marseille.

\begin{abstract}
Considéré comme le fleuron des politiques européennes en matière d'éducation, le programme Erasmus fête ses trente ans en 2017. Si son bilan est présenté comme largement positif pour avoir augmenté les possibilités d'une mobilité estudiantine dans I'Union européenne, la finalité du programme suscite toujours des questions. Dans une « Europe de la connaissance » que l'Union appelle de ses vœux, cette mobilité d'étude n'est pas sans lien avec les marchés du travail et la mobilité professionnelle. Contrairement à l'image d'un étudiant bohème à la recherche de son « identité », les recherches sur la question montrent que l'étudiant Erasmus est souvent davantage un stratège.
\end{abstract}

Depuis plusieurs années dans les titres de journaux fleurit l'idée qu'Erasmus (European Community Action Schema for the Mobility of University Students) aurait créé la « première génération $100 \%$ européenne », "qu’un million de bébés » seraient nés, suite aux rencontres qui se font lors des échanges universitaires $^{1}$. Mais est-ce étonnant que des couples se forment lors des séjours Erasmus, alors que l'homogamie est patente dans un groupe social qui possède de sérieux atouts sociaux? Nous allons voir qu'il est difficile de démêler ce qui tient de la morphologie sociale et scolaire de cette population et ce qui tient des attitudes et/ou des compétences nouvelles, réelles ou supposées, qui auraient été acquises durant le séjour, quand on s'interroge sur les effets de ce programme d'échanges.

La mobilité étudiante, spontanée comme institutionnalisée, a des motifs, des contours, des durées et des issues variables. Face aux nouvelles exigences de flexibilité, de labilité du monde du tra- 
vail, les étudiants et futurs diplômés vont fonder leurs choix (entre filières d'études, entre secteurs d'emploi, entre mobilité et non-mobilité, entre destinations, etc.) sur des ressources socialement distribuées. Le caractère social de ces choix s'exprime aussi par le fait qu'ils dépendent d'opportunités et de contraintes extérieures qui échappent au contrôle des individus,

Le programme Erasmus, regroupé depuis 2014 avec

d'autres dispositifs de mobilité intra-européens sous le chapeau d'Erasmus plus, présente en filigrane

les traces héritées de temps reculés. Ainsi, de la Méditerranée au nord du Vieux continent, l'Europe a, pour assurer sa cohésion, usé des échanges de personnes, d'intellectuels en particulier. même bien dotés et bien nés : "Nous choisissons par rapport à une offre qui ouvre un champ des possibles plus ou moins vaste [...]. Cette offre agit aussi sur nous ${ }^{2}$.»

Étudier les marges, une minorité statistique, comparativement dans plusieurs pays, permet de ne pas se laisser enfermer dans des discours globalisant sur la jeunesse, d'inspiration culturaliste (les jeunes seraient porteurs de mutations généralisées d'un rapport à la mobilité, à la culture, au travail, etc.), sans pour autant oublier des tendances générales, conjoncturelles, des changements qui traversent distinctement les différentes catégories de jeunesse, notamment étudiante.

\section{Le voyage pour études : une mythologie européenne}

L'Europe d'aujourd'hui ne s'est pas construite sur un socle vierge de tout échange, de toute rencontre et de toute migration. Le programme Erasmus, regroupé depuis 2014 avec d'autres dispositifs de mobilité intra-européens sous le chapeau d'Erasmus plus, présente en filigrane les traces héritées de temps reculés. Ainsi, de la Méditerranée au nord du Vieux continent, l'Europe a, pour assurer sa cohésion, usé des échanges de personnes, d'intellectuels en particulier. Tout au long du Moyen Âge, le principal courant migratoire draine vers l'Italie, d'une part, vers Paris, de l'autre, des étudiants des pays de l'Empire et de l'Europe centrale ${ }^{3}$. À cette époque, il ne s'agissait pas d'échanges qui reposaient sur un principe de réciprocité et certains pays étaient plus attractifs que d'autres. Succédera le prestige "du Grand Tour », cette " pérégrination » qui présentera un caractère nouveau, par rapport aux siècles précédents. À côté des études poursuivies, le voyage lui-même, expérience existentielle, sera investi d'une valeur éducative propre ${ }^{4}$. C'est ainsi que l'Europe universitaire des années 1980 a commencé à revendiquer une filiation avec la Renaissance : le programme le plus établi en matière d'échanges universitaires ne fait-il pas allusion au nom latin d'Érasme, un des plus illustres savants de la fin du Moyen Âge qui, né à Rotterdam, étudia à Paris, Oxford et Turin, enseigna le grec à Cambridge et mourût à Bâle ? Pour s'éloigner cependant d'une image romantique de l'étudiant bohème, nous allons observer comment se sont développés les programmes de coopération entre les pays européens. Les migrations, aujourd'hui comme hier, suivent certaines routes construites par des rapports entre nations qui se différencient par leur degré de dynamisme démographique, migratoire et d'ouverture économique et intellectuelle, même si la massification scolaire a rendu l'usage de la mobilité plus visible et que « le trafic » et les réseaux se sont densifiés.

\section{L'institutionnalisation de la mobilité face aux déséquilibres migratoires}

Une politique éducative européenne embryonnaire naît le 9 février 1976. Les ministres européens de l'Éducation réunis décident alors d'un financement de programmes communs d'études, de visites et d'échanges de courte durée. Par la suite, la politique de formation de l'Union européenne va se développer à travers des programmes de mobilité et de coo- 
pération, soit entre les institutions d'enseignement supérieur, soit entre ces dernières et les entreprises. Le programme Erasmus de mobilité dans l'enseignement supérieur, lancé en 1987, a aujourd'hui 30 ans. Il se base sur un principe de réciprocité. Comett, Erasmus et Lingua ${ }^{5}$ sont lancés quant à eux successivement en 1986 et 1989. Ces programmes sont aussi ouverts aux pays de l'Efta (European Free Trade Association), le marché commun. En outre, avec la disparition de l'Union soviétique et les rapides mutations des pays de l'Europe de l'Est, s'ouvrent des perspectives d'une plus grande Europe. La commission met donc en place en 1990 le programme Tempus, pour développer la coopération en éducation avec l'Europe centrale et de l'Est ${ }^{6}$. Ces programmes sont moins connus qu'Erasmus, qui est régulièrement qualifié par des hommes et femmes politiques nationaux et européens comme un programme "populaire », une "success story ». Mais qui concerne-il vraiment?

Même si dans une « Europe de la connaissance » que l'Union appelle de ses vœux, où le niveau moyen de scolarité ne cesse d'augmenter, les flux migratoires sont de plus en plus liés à la mobilité étudiante, celle-ci reste difficile à appréhender ${ }^{7}$. L'Organisation de coopération et de développement économiques (OCDE) en fait une estimation à partir de données sur la population étrangère des universités. Il en ressort que les États européens qui accueillent aujourd'hui le plus d'étudiants étrangers sont dans l'ordre l'Allemagne, la France et la Grande-Bretagne. Plus généralement, selon les données de l'OCDE, la mobilité étudiante internationale reste hautement concentrée dans cinq pays : l'Australie, les ÉtatsUnis, la France, l'Allemagne et le Royaume-Uni.
La mobilité institutionnalisée par le programme Erasmus ne représente qu'environ $10 \%$ de l'ensemble de la mobilité étudiante intra-européenne. La question des conséquences du développement des mobilités dans une Europe où les déséquilibres économiques et migratoires persistent peut difficilement être occultée. Tous les États n'ont pas la même capacité attractive et les étudiants sont bel et bien conscients des rapports de force économiques mondiaux. Malgré le principe de réciprocité, certains pays, comme l'Angleterre, restent des pays essentiellement " importateurs » d'étudiants, alors que d'autres, comme beaucoup de pays du Sud (hormis l'Espagne) et de l'Est de l'Europe, restent des pays essentiellement " expor-
Plus généralement, selon les données de l'OCDE, internationale reste hautement concentrée dans cinq pays : I'Australie, les États-Unis, la France, l'Allemagne et le RoyaumeUni. La mobilité institutionnalisée par le programme Erasmus ne représente qu'environ $10 \%$ de l'ensemble de la mobilité étudiante intra-européenne. la mobilité étudiante maties langagières dans le monde où l'anglais, l'espagnol et le français sont parmi les langues les plus parlées, les pays concernés sont aussi ceux qui attirent le plus d'étudiants Erasmus.

\section{Stratégies transnationales et affinités sélectives entre établissements}

Les stratégies transnationales estudiantines ont en commun de tirer parti de la rareté encore relative des compétences internationales. Contrairement

5. Le Comett (The Community Program for Education Teaching and Training) permet entre autres une coopération régionale et sectorielle entre les institutions d'enseignement supérieur et les entreprises. Lingua concerne l'apprentissage et l'enseignement des langues de l'Europe. 6. Les premiers pays concernés furent la Hongrie, la Pologne, ainsi que les anciennes Tchécoslovaquie et RDA. Il est important de noter que la mobilité avec le programme Tempus, au contraire de celle impulsée par le programme Erasmus, ne doit pas nécessairement impliquer réciprocité. 7. En France, il n'existe pas de système national de recueil systématique de données statistiques concernant les étudiants à l'étranger, ni de dispositif statistique permettant de recenser et de suivre l'évolution de la mobilité internationale des étudiants et des personnels de recherche français. D’autres pays en font une estimation à partir de plusieurs sources. En Italie, on citera par exemple l'AIRE (Anagrafe Italiani Residenti all'Estero), au Royaume-Uni les councils pour le financement de l'enseignement supérieur (HEFCE, HEFCW par exemple), ou encore le Council for International Education. L'immatriculation auprès des services consulaires n'étant dans aucun de ces pays obligatoire, elle ne permet pas de fournir des estimations fiables pour ces populations. Seules les données issues des déclarations des pays membres de l'OCDE et des programmes de mobilité européens permettent d'obtenir une estimation, avec toutes les difficultés que cette mesure comporte, car chaque pays et chaque centre statistique adoptent (comme pour le chômage) ses propres critères pour définir et recenser les étudiants mobiles. 
à la mobilité spontanée qui englobe les étudiants ayant choisi de faire un cursus entier à létranger conduisant à un diplôme, les étudiants Erasmus ne sont que temporairement accueillis dans une université dans laquelle ils sont inscrits comme " étudiants Erasmus ou en échange ». À moins qu'il ne s'agisse de cursus intégrés, létudiant Erasmus, à la fin de ses études, obtient le diplôme de son université d'origine, et non celui de son université d'accueil.

Erasmus est loin de bénéficier à la majorité des étudiants ${ }^{8}$. Les ministres de l'enseignement supérieur de 46 pays européens réunis à Louvain-la-Neuve (Belgique) en 2009 se sont fixés pour objectif de porter la mobilité des étudiants à $20 \%$ au moins, alors que l'objectif n'était que de $10 \%$ à la création du programme Erasmus. Mais les étudiants Erasmus nont jamais constitué beaucoup plus de $5 \%$ des étudiants inscrits dans l'enseignement supérieur européen.

Il existe également une forte inégalité dans la distribution des places entre établissements, filières et disciplines. La répartition Erasmusestloin pour l'ensemble des pays parde bénéficier à la majorité des étudiants. Les ministres de l'enseignement supérieur

de 46 pays européens réunis à Louvain-la-Neuve

(Belgique) en 2009 se sont fixés pour objectif de porter

la mobilité des étudiants

à $20 \%$ au moins, alors que l'objectif n'était que de $10 \%$ à la création du programme Erasmus. ticipants fait apparaître que les plus gros bénéficiaires des échanges sont visiblement les étudiants inscrits dans des disciplines de gestion d'entreprises, "business ", suivis d'assez près des étudiants en langues. Les étudiants de la catégorie ingénierie et technologie et ceux inscrits dans des filières de sciences sociales peuvent être regroupés en troisième position, même si les moyennes peuvent cacher des disparités importantes selon les pays et les institutions d'enseignement supérieur concernés.

Le fait d'être femme n'augmente pas la propension à partir à l'étranger. Un taux de départ féminin sensiblement plus élevé s'explique par les conséquences des choix d'orientation. Quand on isole l'effet propre (" toutes choses égales par ailleurs ») du facteur «femme ", par une modélisation, force est de constater, au sein de chaque filière, que les calculs de proportion de femmes par rapport à l'ensemble des inscrits (hommes et femmes) et leur nombre parmi les étudiants Erasmus partants révèlent des taux similaires.

Le nombre de recherches sur la mobilité des étudiants est allé grandissant ces dernières années. Elles convergent sur le fait que ce public apparaît globalement comme disposant de sérieuses ressources et atouts sociaux ${ }^{9}$. Il nous semble cependant que, de manière générale, une partie des résultats, des recherches françaises pour la plupart, exposés dans l'ouvrage de synthèse de Valérie Erlich laisse trop à penser que les mobilités passagères seraient des formes de "tourisme approfondi » et auraient peu à voir avec d'autres formes de mobilité non encadrées.

L'« acculturation » des étudiants serait-elle tout aussi passagère que leur séjour ${ }^{10}$ ? Nos recherches montrent pourtant que ces mobilités sont prédictives d'autres mobilités. Participent-elles également à la construction de profils européens ou internationaux ? Cette question, que pose Catherine Agulhon en préface de cet ouvrage de synthèse, mériterait d'être posées plus systématiquement et, en effet, la mise en place du programme Erasmus semble avoir donné toute sa consistance au « modèle biographique », à la personnalisation des parcours, dans un enseignement supérieur de plus en plus stratifié. Les discours d'autoréalisation des étudiants en mobilité s'alimentent de l'injonction biographique liée à ce que l'on pourrait décrire comme un changement dans le rapport de l'individu et du social. Cette mutation qui affecte nos sociétés a fait l'objet de nombreuses analyses, dont il ressort qu'il revient à l'individu d'être «l'unité de reproduction de la sphère sociale », pour reprendre la formule d'Ulrich Beck ${ }^{11}$. Ainsi, les individus impute- 
raient à une responsabilité personnelle les conséquences de dépendances et de contraintes extérieures (institutionnelles, sociales, économiques notamment) et seraient tenus de trouver en euxmêmes les ressorts de leur action.

\section{L'expérience Erasmus : le développement personnel au service des études}

Le programme Erasmus, dans sa forme, dans ses modes de sélection, place au centre l'étudiant, plutôt que des équipes pédagogiques «bi-nationales » autour de projets « cognitifs » communs. Il consacre par là même idéologiquement les théories de l'apprentissage qui font du sujet l'acteur de son propre savoir. Parmi les études statistiques sur le programme Erasmus, peu se penchent sur ce que font effectivement les étudiants pendant et après leur séjour à l'étranger. La recherche comparative permet ici de faire la part entre ce qui est inhérent à toute migration et ce qui est plus spécifique à une population donnée, ici étudiante, sélectionnée et relativement privilégiée.

L'université semble être un noyau sans frontières hermétiques, mais qui reste le lieu central de l'expérience des étudiants Erasmus, par le temps qu'ils y passent, par la manière dont ils l'investissent, dont ils la scrutent et l'observent. Un noyau sans frontières, car le séjour Erasmus est aussi investi d'autres désirs, notamment celui du voyage et de la découverte. L'Auberge espagnole ${ }^{12}$, un film qui raconte le séjour d'un jeune étudiant en sciences économiques parti étudier en Espagne pour un an, est assez révélateur et symptomatique des discours et des images présentant l'expérience des étudiants Erasmus à létranger comme ressemblant davantage à un voyage initiatique qu'à un séjour d'études.

Nos recherches montrent cependant que les études ne représentent pas une part marginale de la vie de ces étudiants. De l'analyse lexicale des entretiens transparaissent une richesse et un nombre impor- tant de termes qui se réfèrent aux curricula. Parmi les plus fréquemment employés, on trouve notamment : " cours ", " année ", " temps ", " départements », «professeurs », « niveau », « études », « comprendre », " apprendre », " concours », « examens », « matières », « sciences ». Les divergences de sélectivité des systèmes éducatifs des pays participants permettent également de comprendre l'investissement plus ou moins fort dans le travail et les modes de socia- _ L'université semble être bilité des étudiants Erasmus. Nous avons pu vérifier l'hypothèse qu'il existe bien, au sein de la population étudiée, un certain nombre de pratiques, d'activités qui restent caractéristiques du milieu étudiant. Même si l'intensité des pratiques de loisirs augmente à l'étranger du fait de l'éloignement du foyer familial, les étudiants Erasmus auront des activités propres à leur groupe un noyau sans frontières hermétiques, mais qui reste le lieu central de l'expérience des étudiants Erasmus, par le temps qu'ils y passent, par la manière dont ils l'investissent, dont ils la scrutent et l'observent. Un noyau sans frontières, car le séjour Erasmus est aussi investi d'autres désirs, notamment celui du voyage et de la découverte. d'âge et à leur statut social. Il existe un temps commun à l'ensemble des étudiants Erasmus, qui se démarque assez nettement des temps sociaux des autres catégories de migrants, mais aussi des étudiants "sédentaires » du pays d'accueil et de leurs homologues restés dans les institutions d'enseignement supérieur de leurs pays respectifs. La mobilité institutionnalisée ne présente pas que des avantages financiers, elle réduit aussi énormément les démarches et obstacles administratifs de toutes sortes.

Le vocable autour de l'ouverture et des qualités d'adaptation est très utilisé. Pourtant, leurs comportements en matière de sociabilité sont plutôt conformistes. La distance qu'ils maintiennent avec le pays d'accueil et l'agrégation par nationalité ou zones géographiques habituellement assimilées des étudiants Erasmus n'a rien de bien différent de celles maintenues par un grand nombre d'immigrés des « classes laborieuses. » 
Si on place la focale non plus sur l'individu, son discours, mais sur la formation d'une hiérarchie sociale et scolaire en Europe, force est de constater que les flux migratoires traditionnels et les destinations convoitées ont peu changé, ces dernières décennies, avec l'institutionnalisation de la mobilité étudiante. Les affinités sélectives entre pays et institutions déjà existantes continuent de produire leurs effets.

Chaque destination et chaque institution semble posséder sa propre valence sélective et se voit inscrite dans une hiérarchie. Les institutions les plus ouvertes à l'international sont souvent les plus sélectives. Les inégalités de prestige entre établissements et plus largement entre aires culturelles affectent aussi, pour une part, la morphologie sociale d'une population étudiante Erasmus. Pour simplifier, les échanges entre institutions et départements ne sont pas le fruit du hasard et semblent répondre à l'adage "qui se ressemble s'assemble », ce qui a des conséquences, notamment sur les parcours des diplômés Erasmus.

\section{Caractéristiques sociales des étudiants mobiles et effets sur les parcours}

La structure et la logique du discours sur la mobilité académique ont des effets très concrets sur les comportements des acteurs. La presse relaie abondamment les discours savants ou profanes soulignant que les personnes ayant effectué des séjours d'études à l'étranger s'insèrent plus aisément et occupent plus souvent des postes à responsabilité que ceux qui ont été sédentaires. Mais est-ce la mobilité, le séjour à l'étranger et/ou les caractéristiques sociales et scolaires de cette population qui rendent leur insertion sur le marché du travail plus aisée?

Il existe une surreprésentation significative des catégories sociales favorisées parmi les étudiants Erasmus, et ce de façon d'autant plus nette qu'elle concerne des disciplines où les langues ne font pas partie des enseignements obligatoires. De plus, les Erasmus inscrits dans des filières universitaires non sélectives ont, en moyenne, un parcours scolaire rapide et des compétences migratoires solides résultant d'un passé migratoire riche ${ }^{13}$. Dans beaucoup d'études qui vantent les bienfaits du programme et soulignent la grande satisfaction des participants, ce qui reste lacunaire, c'est bien une analyse des modalités d'insertion des diplômés mobiles sur les marchés du travail.

Il est important, par contre, de s'interroger sur les prédispositions et les contextes socio-économiques variables qui jouent un rôle, au moins indirect, dans un processus d'apprentissage de l'international, et influencent à divers degrés la mobilité professionnelle des Erasmus au cours de leur vie. Chez les étudiants Erasmus, on note, par exemple, qu'immédiatement à leur retour dans leur pays d'origine s'exprime une volonté quasi-unanime de "repartir ». Les motifs se situent dans le désir de voyage, la poursuite des études ou la recherche de travail « ailleurs » ou pour " ne pas revenir en arrière », selon les expressions des personnes interrogées ${ }^{14}$. Le séjour Erasmus est ainsi un tremplin vers d'autres mobilités, qui prennent cependant des formes bien différentes selon les appartenances socio-nationales. Les étudiants italiens interrogés dans nos enquêtes, issus de filières non sélectives des universités, souhaitent souvent, une fois diplômés, commencer une carrière à l'étranger. Ceci traduit bien la difficulté à réimporter chez soi les ressources sociales liées au statut d'étudiants privilégiés dans un autre pays, car dégagé de certains problèmes financiers et/ou de positionnement social ${ }^{15}$.

\section{Des études à l'emploi : des inégalités de trajectoire et d'insertion professionnelle}

Pour prendre l'exemple de l'Italie, la composition sociale de l'émigration a certes changé depuis la fin 
de la Seconde Guerre mondiale, mais faut-il pour autant conclure à une disparition de la contrainte ? Le poids des structures sociales n'est pas amoindri, ce sont les formes des pressions, des injonctions qui se sont transformées. À des flux massifs d'émigrés antérieurs aux années 1960, se substituent en Italie des flux quantitativement moins importants, mais socialement significatifs. Finalement, la mobilité étudiante est la face positive des migrations. Au fur et à mesure que le nombre de qualifiés du supérieur augmente, la composition des flux migratoires se transforme. Les choix opérés par les étudiants Erasmus restent ainsi étroitement liés à leurs perspectives d'emploi au sortir du système universitaire, qui sont elles-mêmes extrêmement sensibles à létat général des segments des marchés nationaux du travail. L'aspiration au cosmopolitisme, à l'international, correspond à une volonté d'ascension sociale (lélite lui étant souvent associée). Comme dans l'enseignement supérieur, la croissance quantitative, l'augmentation du nombre d'étudiants mobiles ne garantit pas, en soi, la démocratisation de l'accès et de la réussite pour tous dans le monde professionnel. Certains enfants de catégories populaires et moyennes qui ont " réussi » scolairement, dans des segments massifiés de l'Enseignement supérieur, dont on méprise le «localisme ", se trouvent aujourd'hui légitimement attirés par « l'international » qui procure une certaine reconnaissance sociale. Mais ils se trouvent aussi confrontés à la recomposition sociale des migrations et des rapports de force.

L'insertion professionnelle des étudiants de milieux défavorisés sortant des études supérieures, étudiée par Paul Pasquali, faite de "petits boulots », d'alternance et là aussi de voyage à l'étranger parfois, montre que cette catégorie sociale, malgré sa mobilité, se heurte à la compétition d'autres diplômés d'autres milieux sociaux ${ }^{16}$. En définitive, l'amplitude et les modalités du déplacement social dépendent surtout de l'état des marchés scolaires et du travail qualifié, comme du soutien des proches. On peut, au passage, saluer la volonté de
Paul Pasquali d'éviter l'écueil des débats sur l'existence d'une corrélation entre mobilité géographique et/ou sociale et pathologie. De nombreuses recherches ne résistent pas à ce penchant pour les questions de souffrance, « d'identité » qui seraient alors devenues incohérentes par le déplacement.

Au-delà des mythes, il n'est pas possible de conjuguer Au-delà des mythes, il n’est aujourd'hui l'expérience pas possible de conjuguer étudiante de mobilité en aujourd'huil'expérience Europe au singulier. Choi- étudiante de mobilité sir ses références, sa ou en Europe au singulier. ses langues, ses identifica- Choisir ses références, tions, comme son emploi, sa ou ses langues, ses sont des possibilités iné- identifications, comme son galement réparties au sein emploi, sont des possibilités des populations étudiantes inégalement réparties au sein et mobiles. Les fortes dis- des populations étudiantes parités, toujours existantes et mobiles en Europe, ne sont pas sans conséquences sur un programme dont les principes de coopération et de réciprocité se heurtent aux principes de compétition et de concurrence qui existent partout ailleurs. Nous souhaiterions conclure sur ce point en s'associant aux études qui soulignent qu'il manque encore aujourd'hui beaucoup de données fiables permettant de comparer mobilité organisée et mobilité non encadrée, mobilité étudiante et mobilité professionnelle, ainsi que plus généralement des recherches sur les migrations qualifiées en Europe.

\section{Conclusion}

L'homogénéisation supposée croissante autour d'une culture plus ou moins mondialisée ne suffit pas pour que s'établissent spontanément des échanges égalitaires entre des institutions issues d'histoires diverses ou entre des groupes sociaux éloignés. La question de la mobilité institutionnalisée en Europe ne peut être discutée sans prendre en compte la question sociale. 
Le fait qu'Erasmus plus renforce aujourd'hui les relations entre les entreprises, les organismes socio-professionnels et les établissements d'enseignement supérieur n'a rien d'anodin non plus et ne peut être sans conséquences sur les liens déjà existants entre mobilité étudiante et mobilité professionnelle. En 2011-2012, un étudiant Erasmus sur cinq (soit près de 50000 au total) a choisi l'option du stage en entreprise, un pourcentage en croissance continue depuis. Contrastant avec l'enthousiasme d'un brain movement en vogue, qui se traduit notamment par l'expansion des échanges universitaires en Europe, nous avons voulu rappeler ici que, même si les étudiants sont certes globalement très satisfaits de leurs expériences à l'étranger ${ }^{17}$, les jeunes sta- giaires, diplômés de l'enseignement supérieur que nous avons rencontrés, qui débutent leur carrière à l'étranger, ne vivent pas toujours la mobilité, lorsqu'elle se transforme en émigration ou en précarité, de façon positive. À qui profite alors le développement des migrations étudiantes et professionnelles en Europe ? N'existerait-il pas un réel déséquilibre des bénéfices? Dans ce contexte, il convient de s'interroger sur les conséquences de l'ensemble des politiques migratoires en Europe, dont le programme Erasmus constitue peu ou prou un révélateur, a fortiori quand les disparités de développement économique, social et politique au sein de l'Union européenne augmentent par l'arrivée de nouveaux États membres d'Europe centrale et orientale. 